Pacific Journal of Mathematic 


\title{
UNIFORM CONTINUITY OF CONTINUOUS FUNCTIONS OF METRIC SPACES
}

\author{
MASAhiko Atsuji
}

In this paper we intend to find equivalent conditions under which continuous functions of a metric space are always uniformly continuous. Isiwata has attempted to prove a theorem in a recently published paper [3] by a method that has a close relation with ours. Unfortunately he does not accomplish his purpose, so we shall give a correct theorem (Theorem 3) in the last part of this paper and, for this purpose, give a condition for the existence of a uniformly continuous unbounded function in a metric space (Theorem 2).

In this paper the space $S$, unless otherwise specified, is the metric space with a distance function $d(x, y)$, and, for a positive number $\alpha$, the $\alpha$-sphere about a subset $A\{x ; d(A, x)<\alpha\}$ is denoted by $S(A, \alpha)$; the function is the real valued continuous mapping.

DEFINITION 1. Let us consider a family of neighborhoods $U_{n}$ of $x_{n}$ such that $\left\{x_{n}\right\}$ is a sequence of distinct points and $U_{m} \cap U_{n}=\phi$ (=empty) for $m \neq n$. Let $f_{n}(x)$ be a function such that $f_{n}\left(x_{n}\right)=n$ and $f_{n}(x)=0$ for $x \notin U_{n}$. Then a mapping constructed from the family is a mapping $f(x)$ defined by $f(x)=f_{n}(x)$ for $x$ belonging to some $U_{n}$ and $f(x)=0$ for the other $x$.

Lemma. Consider a family of neighborhoods $U_{n}$ of $x_{n}$ satisfying the following conditions :

(1) $\left\{x_{n}\right\}$, which consists of distinct points, has no accumulation point,

(2) $\bar{U}_{m} \cap \bar{U}_{n}=\phi, m \neq n(\bar{U}$ a closure of $U)$, and $U_{n} \subset S\left(x_{n}, 1 / n\right)$,

(3) there is a sequence of points $y_{n}$ such that distances of $x_{n}$ and $y_{n}$ converge to 0 and $y_{n}$ does not belong to any $U_{m}$; then the mapping constructed from the family is continuous and not uniformly continuous. When $\left\{x_{n}\right\}$ is a sequence containing infinitely many distinct points and has no accumulation point, there is a family of neighborhoods of $x_{n}$ satisfying (2); if $\left\{x_{n}\right\}$ further contains infinitely many distinct accumulation points, then the family besides satisfies (3).

Proof. The continuity of the mapping constructed from the family follows from $\cup U_{n_{i}}=\cup \bar{U}_{n_{i}}$ for any subsequence $\left\{n_{i}\right\}$ of indices; the mapping is not uniformly continuous by (3). Suppose $\left\{x_{n}\right\}$ consists of distinct accumulation points and has no accumulation point, then, by an inductive process, we have neighborhood $V_{n}$ of $x_{n}$ such that $V_{n}$ $\subset S\left(x_{n}, 1 / n\right)$ and $\bar{V}_{m} \cap \bar{V}_{n}=\phi$, and have $y_{n}$ and a neighborhood $U_{n}$ of $x_{n}$

Received May 24, 1957. In revised form September 6, 1957. 
such that $U_{n} \nexists y_{n} \in V_{n}, U_{n} \subset V_{n}$.

DEFINITION 2. Let $x$ be isolated in a metric space, then we write $I(x)$ for a supremum of positive numbers $\alpha$ such that $S(x, \alpha)$ consists of $x$ alone.

THEOREm 1. The following conditions on a metric space $S$ are equivalent

(1) If $\left\{x_{n}\right\}$ is a sequence of points without accumulation point, then all but finitely many members of $x_{n}$ are isolated and inf $I\left(x_{n}\right)$ for the isolated points is positive.

(2) If a subset $A$ of $S$ has no accumulation point then all but finitely many points of $A$ are isolated and inf $I(x)$ for all the isolated points of $A$ is positive.

(3) The set $A$ of all accumulation points in $S$ is compact and inf $I\left(x_{n}\right)$ is positive for any sequence $\left\{x_{n}\right\}$ in $S-A$ which has no accumulation point (Isiwata [2], Theorem 2).

(4) $\bar{A} \cap \bar{B}=\phi$ implies $S(A, \alpha) \cap S(B, \alpha)=\phi$ for some $\alpha$ (Nagata [4], Lemma 1).

(5) $\bigcap_{n=1}^{\infty} \bar{A}_{n}=\phi$ implies $\bigcap_{n=1}^{\infty} S\left(A_{n}, \alpha\right)=\phi$ for some $\alpha$.

(6) For any function $f(x)$, there is a positive integer $n$ such that every point of $A=\{x ;|f(x)| \geqq n\}$ is isolated and $\inf _{x \in A} I(x)$ is positive.

(7) All functions of $S$ are uniformly continuous.

(8) All continuous mappings of $S$ into an arbitrary uniform space $S^{\prime}$ are uniformly continuous.

Proof. Since the equivalence of (1) and (3) is simple, we shall show $(1) \rightarrow(8) \rightarrow(7) \rightarrow(6) \rightarrow(5) \rightarrow(4) \rightarrow(2) \rightarrow(1)$.

$(1) \rightarrow(8)$ : If a continuous mapping $f(x)$ of $S$ is not uniformly continuous, there is an "entourage" $V$ (in the sense of Bourbaki) of $S^{\prime}$ such that $d\left(x_{n}, y_{n}\right)<1 / n$ and $\left(f\left(x_{n}\right), f\left(y_{n}\right)\right) \notin V$ for any positive integer $n$ and for some $x_{n}$ and $y_{n} . \quad\left\{x_{n}\right\}$ contains infinitely many distinct points. If $\left\{x_{n}\right\}$ has an accumulation point $x$, there are subsequences $\left\{x_{n_{i}}\right\}$ and $\left\{y_{n_{i}}\right\}$ of $\left\{x_{n}\right\}$ and $\left\{y_{n}\right\}$ converging to $x$, and, since $f(x)$ is continuous, $(f(x)$, $\left.f\left(x_{n_{i}}\right)\right) \in W$ and $\left(f(x), f\left(y_{n_{i}}\right)\right) \in W$ for $W$ satisfying $W \cdot W \subset V$ (we may assume $\left.W^{-1}=W\right)$ and for all sufficiently large $i$. Hence we have $\left(f\left(x_{n_{i}}\right)\right.$, $\left.f\left(y_{n_{i}}\right)\right) \in V$, which is excluded. Consequently $\left\{x_{n}\right\}$ hes no accmulation point and inf $I\left(x_{n}\right)=r>0$ for all sufficiently large $n$, which contradicts the first inequality of $f$ for $\mathrm{n}$ satisfying $r>1 / n$.

$(8) \rightarrow(7)$ is obvious.

$(7) \rightarrow(6)$ : If, for some function $f(x)$ and every $n$, there is an accumulation point $x_{n}$ such that $\left|f\left(x_{n}\right)\right| \geqq n,\left\{x_{n}\right\}$ contains infinitely many distinct elements and has no accumulation point, then, by the Lemma, we have 
a function which is not uniformly continuous. Suppose that every point of $A=\{x ;|f(x)| \geqq n\}$ is isolated and inf $I(x)=0$. Then there is a sequence $\left\{x_{n}\right\}$ in $A$ such that inf $I_{n}=0, I_{n}=I\left(x_{n}\right) . \quad\left\{x_{n}\right\}$ has no accumulation point, and we may assume $I_{n}<1 / n$. If distances of distinct points of $\left\{x_{n}\right\}$ are greater than a positive number $e$, then, for all $n$ satisfying $e>4 I_{n}, \quad x_{n}$ and $y_{n}\left(\neq x_{n}, \in S\left(x_{n}, 2 I_{n}\right)\right)$ satisfy the conditions of the Lemma. In the other case, there are arbitrarily large $m$ and $n$ satisfying $d\left(x_{m}, x_{n}\right)<e$ for any positive number $e$, and we have, by an inductive process, a subsequence $\left\{y_{i}\right\}$ of $\left\{x_{n}\right\}$ satisfying $d\left(y_{2 i-1}, y_{2 i}\right)<1 / i$. Then $y_{2 i-1}$ and $y_{2 i}$ satisfy the conditions of the Lemma.

$(6) \rightarrow(5)$ : Let $\cap S\left(A_{n}, 1 / m\right) \neq \phi$ for every $m$ in spite of $\cap \bar{A}_{n}=\phi$. We have a point $x_{1}$ contained in $\bigcap_{n} S\left(A_{n}, 1\right)$ and a point $y_{1}$ distinct from $x_{1}$ satisfying $d\left(x_{1}, y_{1}\right)<1$. Suppose $B_{i}=\left\{x_{1}, \cdots, x_{i}\right\}$ consists of distinct points such that $x_{j} \in \cap S\left(A_{n}, 1 / j\right), x_{j}$ and $y_{j}$ are distinct and $d\left(x_{j}, y_{j}\right)$ $<1 / j, j=1, \cdots, i$. Since, for any point $x, \cap_{n} S\left(A_{n}, 1 / m\right)$ does not contain $x$ for a sufficiently large $m, \cap S\left(A_{n}, 1 /(i+1)\right)$ contains a point $x_{i+1}$ being not contained in $B_{i}$, and some $A_{n}$ contains $y_{i+1}$ distinct from $x_{i+1}$ satisfying $d\left(x_{i+1}, y_{i+1}\right)<1 /(i+1)$. Thus we have a sequence $\left\{x_{n}\right\}$ of distinct points and $\left\{y_{n}\right\}$ such that $x_{m} \in \cap S\left(A_{n}, 1 / m\right), x_{n}$ and $y_{n}$ are distinct, and $d\left(x_{n}, y_{n}\right)<1 / n . \quad\left\{x_{n}\right\}$ has no accumulation point because of $\cap \bar{A}_{n}=\phi$. The function obtained from the Lemma does not satisfy the condition (6) whether all but finitely many members of $x_{n}$ are isolated or not. $(5) \rightarrow(4)$ is obvious.

$(4) \rightarrow(2)$ : Suppose $A$ has infinitely many accumulation points $x_{n}, n=$ $1,2, \cdots$. Since $B=\left\{x_{n}\right\}$ has no accumulation point, there is a sequence $C=\left\{y_{n}\right\}$ having no accumulation point such that $d\left(x_{n}, y_{n}\right)<1 / n, B \cap C=\phi$. $\bar{B} \cap \bar{C}=B \cap C=\phi$, and $S(B, \alpha) \cap S(C, \alpha)=\phi$ for no $\alpha$. If every point of $A$ is isolated and $\inf I(x)=0$, we have a sequence $\left\{x_{n}\right\}$ such that lim $I\left(x_{n}\right)=0$, and have a sequence $\left\{y_{n}\right\}$ with the same properties as the above.

$(2) \rightarrow(1)$ is obvious.

Recently Isiwata has stated a theorem ([3], Theorem 4) which is related to our Theorem 1. However the first step in his proof is wrong. We shall give a correct form of the theorem in Theorem 3. Let us first give a counterexample for the statement "In a connected metric space which is not totally bounded, there exists a sequence $\left\{x_{n}\right\}$ and a uniformly continuous function $f$ such that $f\left(x_{n}\right)=n$ '.

EXAmPle. Denoting the points of the plane by polar-coordinate, 
we consider the following subsets of the plane:

$$
\begin{aligned}
A_{m} & =\{(r, \theta) ; 0 \leqq r \leqq 1, \theta=\pi / m\}, \\
S & =\bigcup_{m=1}^{\infty} A_{m} .
\end{aligned}
$$

We define the distance of the points of $S$ by

$$
\begin{aligned}
d\left((r, \theta),\left(r^{\prime}, \theta^{\prime}\right)\right) & =\left|r-r^{\prime}\right| & & \text { as } \theta=\theta^{\prime} \text { or } r r^{\prime}=0, \\
& =r+r^{\prime} & & \text { as } \theta \neq \theta^{\prime},
\end{aligned}
$$

then $S$ is obviously a connected metric space which is not totally bounded. When $f(x), x \in S$, is a uniformly continuous function of $S$, there is a positive integer $n$ such that $d(x, y)<1 / n$ implies $|f(x)-f(y)|$ $<1$. If $x$ is contained in $A_{m}$, there are points $y_{0}=0=$ pole, $y_{1}, \cdots, y_{r}$ $=x, r \leqq n+1$, of $A_{m}$ such that $d\left(y_{i-1}, y_{i}\right)<1 / n, i=1, \cdots, r$.

$$
|f(0)-f(x)| \leqq\left|f(0)-f\left(y_{1}\right)\right|+\cdots+\left|f\left(y_{r-1}\right)-f(x)\right| \leqq n+1 ;
$$

namely $f(x)$ is bounded.

Definition 3. Let $e$ be a positive number, then the finite sequence of points $x_{0}, x_{1}, \cdots, x_{m}$ satisfying $d\left(x_{i-1}, x_{i}\right)<e, i=1, \cdots, m$, is said to be an e-chain with length $m$. If, for any positive number $e$, there are finitely many points $p_{1}, \cdots, p_{i}$ and a positive integer $m$ such that any point of the space can be bound with some $p_{j}, 1 \leqq j \leqq i$, by an $e$-chain with length $m$, then the space is said to be finitely chainable.

THEOREM 2. A metric space $S$ admits a uniformly continuous unbounded function if and only if $S$ is not finitely chainable.

Proof. Verification of "only if" part is analogous to that stated in the above example, hence is passed over. Let $S$ be not finitely chainable, then there is a positive number $e$ such that, for any finitely many points and a positive integer $n$, there is a point which cannot be bound with any one of points selected above by an $e$-chain with length $n$. We denote by $A_{0}^{n}$ the set of all points which can be bound with a fixed $x_{0}$ by an $e$-chain with length $n$.

(1) When $A_{0}^{n} \neq A_{0}^{n+1}$ for every $n$, we put

$$
f(x)=(n-1) e+d\left(x, A_{0}^{n-1}\right)
$$

for $x$ belonging to $A_{0}^{n}$ and not to $A_{0}^{n-1}$, and $f(x)=0$ for $x \notin A_{0}=\cup A_{0}^{n}(f(x)$ $=d\left(x_{0}, x\right)$ for $\left.x \in A_{0}^{1}\right)$. Since $S\left(A_{0}, e\right)=A_{0}, f(x)$ is uniformly continuous on $S$ if it is so on $A_{0}$. Let $A_{0}^{n} \ni x \notin A_{0}^{n-1}$ and $d(x, y)<e^{\prime}<e$, then $A_{0}^{n+1} \ni y \notin A_{0}^{n-2}$. (i) When $y$ is in $A_{0}^{n-1}$, then 


$$
f(y)=(n-2) e+d\left(y, A_{0}^{n-2}\right)
$$

and $d\left(x, A_{0}^{n-1}\right)<e^{\prime}, d\left(y, A_{0}^{n-2}\right)<e$, hence $f(y) \leqq f(x)$. If $d\left(y, A_{0}^{n-2}\right)<e-e^{\prime}$, then $d\left(y, y^{\prime}\right)<e-e^{\prime}$ for some $y^{\prime}$ of $A_{0}^{n-2}$ and $d\left(x, y^{\prime}\right) \leqq d(x, y)+d\left(y, y^{\prime}\right)<e$, so that $x$ is in $A_{0}^{n-1}$, which is excluded. Therefore $d\left(y, A_{0}^{n-2}\right) \geqq e-e^{\prime}$ and

$$
\begin{aligned}
|f(x)-f(y)| & =f(x)-f(y)=e+d\left(x, A_{0}^{n-1}\right)-d\left(y, A_{0}^{n-2}\right) \\
& <e+e^{\prime}-\left(e-e^{\prime}\right)=2 e^{\prime} .
\end{aligned}
$$

(ii) When $y$ is in $A_{0}^{n}$ and not in $A_{0}^{n-1}$, then

$$
f(y)=(n-1) e+d\left(y, A_{0}^{n-1}\right),
$$

and we have

$$
|f(x)-f(y)|=\left|d\left(x, A_{0}^{n-1}\right)-d\left(y, A_{0}^{n-1}\right)\right| \leqq d(x, y)<e^{\prime}
$$

(cf. the proof of Prop. 3 of $\S 2,[1]$ ). (iii) The remaining case for $y$ is similar to (i). Consequently $f(x)$ is uniformly continuous on $A_{0}$.

( 2 ) When $A_{0}^{n}=A_{0}^{n+1}$ for some $n$, then $A_{0}^{m}=A_{0}^{n}$ for every $m \geqq n$, and, in the similar way to (1), $A_{1}=\cup A_{1}^{n}$ is obtained from a point of $S-A_{0}$. If we can make an unbounded function which is uniformly continuous on $A_{1}$, our proof will be complete.

(3) When we cannot, for every $m(0 \leqq m \leqq n)$, construct a desired function on $A_{m}$ obtained in the same way as (2), $A_{0}, \cdots, A_{n}$ cannot cover the space, because the space is not finitely chainable; namely we have a sequence of infinitely many subsets $A_{0}, A_{1}, \ldots$ when our proof is not complete in the similar way to (2). Then we put $f(x)=n$ for $x$ of $A_{n}$ and $f(x)=0$ for $x$ which is not in any $A_{n}$. Then, since $S\left(A_{m}, e\right) \cap A_{n}=\phi$ for any $m \neq n$ and $S\left(\cup A_{n}, e\right)=\cup A_{n}, f(x)$ is uniformly continuous.

THEOREM 3. If $S$ is a connected metric space which is not finitely chainable, then the set of all uniformly continuous functions of $S$ does not form a ring.

Proof. The following verification is essentially due to Isiwata [3]. There is, by Theorem 2, a uniformly continuous unbounded function $f(x)$ of the space, and we have a sequence $A=\left\{x_{n} ; n=1,2, \cdots\right\}$ such that $f\left(x_{n}\right)=a_{n}, \quad a_{n+1}-a_{n} \geqq 1, a_{1} \geqq 1 ; A$ has no accumulation point. For some positive number $\alpha, d(x, y)<\alpha$ imlies $|f(x)-f(y)|<1 / 3$, and so $S\left(x_{m}\right.$, $\alpha) \cap S\left(x_{n}, \alpha\right)=\phi$ for $m \neq n$. We put

$$
h(x)=1-d(A, x) / \alpha \quad \text { and } \quad G=\bigcup_{n} S\left(x_{n}, \alpha\right)
$$




$$
f^{\prime}(x)= \begin{cases}h(x) & \text { for } x \in G, \\ 0 & \text { for } x \notin G .\end{cases}
$$

$h(x)$ is uniformly continuous on the space, because $d(A, x)$ is so (cf. Prop. 3 of $\S 2,[1]) . \quad h(x)>0$ and $h(y) \leqq 0$ for $x$ of $G$ and $y$ of $S-G$ respectively, so we have

$$
|h(x)-h(y)|=h(x)-h(y) \geqq h(x)=\left|f^{\prime}(x)-f^{\prime}(y)\right| .
$$

Hence $f^{\prime}(x)$ is uniformly continuous on the space. $g(x)=f(x) f^{\prime}(x)$ is not uniformly continuous. In fact, if it is uniformly continuous, $d(x, y)<\beta$ implies

$$
|g(x)-g(y)|<1 \quad \text { and } \quad|f(x)-f(y)|<1
$$

for some $\beta(\leqq \alpha)$. We select a positive integer $n$ such that $a_{n}$ is greater than $1+4 \alpha / \beta$, and take a point $y$ such that $\beta / 2 \leqq d\left(x_{n}, y\right)<\beta$ (it is possible to take such a point because of the connectedness of the space). Then, by $(*)$, we have $\left|a_{n}-f(y)\right|<1, f(y)>a_{n}-1 \geqq 0$, and

$$
\begin{aligned}
\left|g\left(x_{n}\right)-g(y)\right| & =\left|a_{n}-(1-d(A, y) / \alpha) f(y)\right|=\left|a_{n}-f(y)+d\left(x_{n}, y\right) f(y) / \alpha\right| \\
& \geqq\left|d\left(x_{n}, y\right) f(y) / \alpha\right|-\left|a_{n}-f(y)\right|>d\left(x_{n}, y\right) f(y) / \alpha-1 \\
& >\beta\left(a_{n}-1\right) / 2 \alpha-1>\beta(1+4 \alpha / \beta-1) / 2 \alpha-1=1,
\end{aligned}
$$

which contradicts $(*)$.

\section{REFERENCES}

1. N. Bourbaki, Topologie générale, Chap. IX, Paris Hermann, 1948.

2. T. Isiwata, On uniform continuity of $C(X)$ (Japanese), Sugaku Kenkyu Roku of Tokyo Kyoiku Daigaku 2 (1955), 36-45.

3. _._. Structures of a uniform space $X$ and $C(X)$, Sci. Rep. Tokyo Kyoiku Daigaku 5 (1956), 174-184.

4. J. Nagata, On the uniform topology of bicompactifications, J. Inst. Polytech. Osaka City Univ. 1 (1959), 28-38.

KANAZAWA JAPAN 


\section{PACIFIC JOURNAL OF MATHEMATICS}

\section{EDITORS}

David Gilbarg

Stanford University

Stanford, California

R. A. Beaumont

University of Washington

Seattle 5 , Washington
A. L. Whiteman

University of Southern California Los Angeles 7, California

E. G. Straus

University of California

Los Angeles 24, California

\section{ASSOCIATE EDITORS}
E. F. BECKENBACH
C. E. BURGESS
M. HALL
E. HEWITT
A. HORN
V. GANAPATHY IYER
R. D. JAMES
M. S. KNEBELMAN

L. NACHBIN

I. NIVEN

T. G. OSTROM

H. L. ROYDEN
M. M. SCHIFFEI

G. SZEKERES

F. WOLF

K. YOSIDA

\section{SUPPORTING INSTITUTIONS}

UNIVERSITY OF BRITISH COLUMBIA

CALIFORNIA INSTITUTE OF TECHNOLOGY

UNIVERSITY OF CALIFORNIA

MONTANA STATE UNIVERSITY

UNIVERSITY OF NEVADA

OREGON STATE COLLEGE

UNIVERSITY OF OREGON

OSAKA UNIVERSITY

UNIVERSITY OF SOUTHERN CALIFORNIA
STANFORD UNIVERSITY

UNIVERSITY OF TOKYO

UNIVERSITY OF UTAH

WASHINGTON STATE COLLEGE

UNIVERSITY OF WASHINGTON

AMERICAN MATHEMATICAL SOCIETY

CALIFORNIA RESEARCH CORPORATION

HUGHES AIRCRAFT COMPANY

THE RAMO-WOOLDRIDGE CORPORATION

Printed in Japan by Kokusai Bunken Insatsusha

(International Academic Printing Co., Ltd.), Tokyo, Japan 


\section{Pacific Journal of Mathematics}

\section{Vol. 8, No. 1 \\ March, 1958}

Shimshon A. Amitsur, Commutative linear differential operators ......... 1

Masahiko Atsuji, Uniform continuity of continuous functions of metric

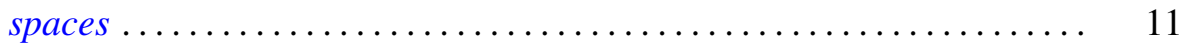

S. P. Avann, A numerical condition for modularity of a lattice . . . . . . . . . 17

Raymond G. D. Ayoub, A mean value theorem for quadratic fields........ 23

Errett Albert Bishop, Subalgebras of functions on a Riemann surface ..... . 29

Shaul Foguel, The relations between a spectral operator and its scalar

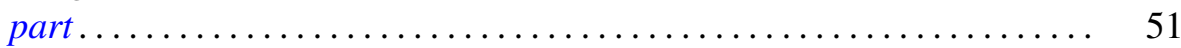

John Rolfe Isbell, Euclidean and weak uniformities ................. 67

Samuel Karlin and James L. McGregor, Many server queueing processes with Poisson input and exponential service times .............. 87

Paul Joseph Kelly and Ernst Gabor Straus, Curvature in Hilbert geometries....................................... 119

John W. Lamperti, Stationary measures for certain stochastic processes . . . 127

Richard Scott Pierce, Distributivity and the normal completion of Boolean

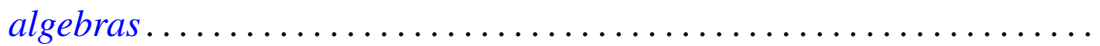

F. M. Ragab, Transcendental addition theorems for the hypergeometric

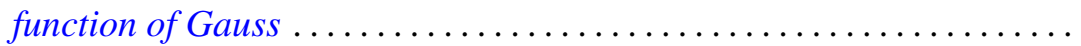

William T. Reid, Principal solutions of non-oscillatory self-adjoint linear differential systems ................................ 147

Maurice Sion, On general minimax theorems .................... 171

Chien Wenjen, On semi-normed ${ }^{*}$-algebras .................... 177 